\title{
A microfluidic-based PDMS prism for SPR imaging system
}

\author{
Lulu Zhang ${ }^{1}$, Xing Chen ${ }^{1}$, Haoyuan Cai ${ }^{1}$, Hui Li ${ }^{1}$, Jianhai Sun ${ }^{1}$, Dafu Cui ${ }^{1}$, Chaoya Peng ${ }^{2}$ \\ ${ }^{1}$ State key laboratory of transducer technology, Institute of Electronics, Chinese Academy of \\ Sciences, Beijing 100190, China \\ Corresponding author: chenxing2004star@yahoo.com.cn \\ ${ }^{2}$ Institute of Microbiology, Chinese Academy of Sciences, Beijing 100190, China
}

\begin{abstract}
:
Surface plasmon resonance (SPR) is a versatile technique for label-free, high sensitive, real time detection of biomolecular binding events. The conventional SPR sensors using glass prisms, matching oil and flow cell require complex operation steps which are more suitable for a laboratory study. PDMS prism abolishes using the index matching oil or film and increases the consistency of reaction for the gold film was sputtered on it directly and it is much easier to integrate with microfluidic channels. In this paper, we developed a low-cost, convenient SPR imaging system with a microfluidic-based PDMS prism. The simulation and experiment results of imaging SPR resonant angle in the air matched with each other. We detected the air and gases breathed out by different persons that indicate the SPR imaging system have a good sensitivity to distinguish different samples.
\end{abstract}

Key words: PDMS prism, Surface plasmon resonance (SPR), imaging, microfluidic

\section{Introduction}

Surface plasmon resonance (SPR) is a powerful analytical method for the study of biomolecular interactions at the transducer surfaces [1-3]. It is considered as a good alternative to traditional analytical techniques, owing to the advantages of rapid and real-time analysis without labels. In principle, the SPR phenomenon is excited by the evanescent wave of the incident light at the interface of a thin metallic film and a dielectric. SPR is highly sensitive to changes in the refractive index close to metal surfaces. In recent years, various approaches have been developed to realize the SPR sensors, such as prism coupler, grating coupler, optical waveguide coupler. The prismtype attenuated total reflection (ATR) method owes the highest sensitivity and it has been commercialized by several companies. However, commercialized SPR instruments that use glass prism as coupler are more suitable for a laboratory study for its high cost and complex operation steps.

Poly(dimethylsiloxane) (PDMS) is becoming more popular in microfluidic technology owing to its flexibility, low cost, easy-to-fabrication and rapid sealing properties. Due to its unique properties, this material has been used for a large variety of systems, as could be electrochemical biosensor, SPR sensor, cytometry [4-6]. Llobera developed a high integration micro-optical element by hollow PDMS prism[7]. PDMS prism if used in imaging SPR sensors abolishes using the index matching oil or film and increases the consistency of reaction. What's more, it is much easier to integrate with microfluidic channels[8] for low-cost high performance rugged field use.

In this paper, we developed a low-cost, convenient SPR imaging system with a microfluidic-based PDMS prism [9].

\section{Experimental and Fabrication The scheme of the SPR imaging system}

The reported SPR imaging sensor is based on prism coupling mode of the Kretschmann structure shown in Fig.1. The sensor consists of a red laser light source $(650 \mathrm{~nm}$ peak wavelength), a PDMS prism of equilateral triangle shape with gold (50nm thick) coated on one surface, an objective lens and a CCD camera. The laser emits a parallel light beam that passes through the prism and strikes the sensor surface. And then the beam of light reflected into the CCD detector. The laser and the polarizing filter and lens system are installed on a circumrotated arm. The CCD with a higher resolution is immobilized on another circumrotated arm. Both circumrotated arms are controlled by the stepper motor and the coding 
system. The incident angle changed from 40 to 70 degree and the incident angle range satisfies the needs for gas and liquid experiment. The resonant angle is very sensitive to changes in the refractive index of the medium adjacent to the metal layer supporting surface plasmons. Therefore, variations in the refractive index at the sensor surface induced by the interaction between target analyte molecule and biomolecular recognition element immobilized on the sensor surface may be observed as changes in the resonant angle.

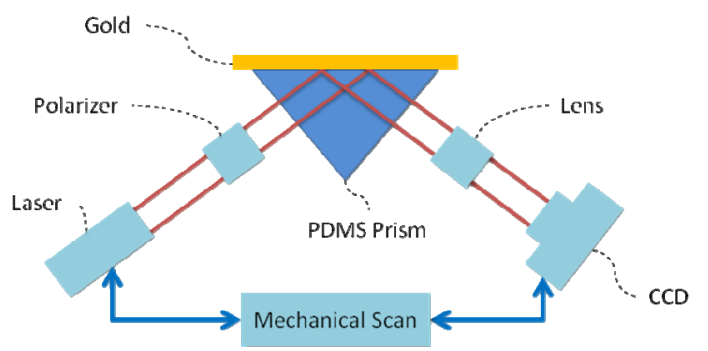

Fig. 1. Schematic of PDMS based SPR imaging system

\section{SPR Flow cell}

Gas or Liquid sample flows over the sensor surface through a hard-soft flow cell which consists of one rigid, CNC-machined PMMA base, one flexible PDMS microfluidic layer, and metal pipes. The PDMS layer has four parallel microfluidic channels, that each channel is $2 \mathrm{~mm}$ wide, and can reversibly adhere to SPR gold chip for forming a sealed microfluidic network. This microfluidic flow cell does not only keep the original advantage of conventional PDMSbased flow cell such as the intrinsically soft interface, easy-to-fabrication, and low cost, but also has a rigid, robust, easy-to-use interface to tubing connection.

\section{The fabrication of the PDMS prism}

The fabrication procedure of a PDMS prism was as follows. A 10:1 or 6:1 mixture of PDMS oligomer and crosslinking agent (Sylgard 184), which had been degassed under vacuum, was poured into the silanized assembled glass mould, and then cured in an oven at $80{ }^{\circ} \mathrm{C}$ for 1 h. After the silanized assembled glass mould was easily removed, a PDMS prism was achieved. The PDMS prisms were then coated with $2 \mathrm{~nm}$ of chromium then $50 \mathrm{~nm}$ of gold film by magnetron sputtering technology.

\section{Results}

\section{The SPR response with the PDMS prism}

We have simulated the SPR resonant angle in the air by Winspall software. The refractive index(RI) of the PDMS is 1.41 , and the gold $n=$
0.1617, $k=3.2118$. The simulation resonant angle was 43.7 degree. We also scanned the incident angles by our SPR imaging system, and the resonant angle is 45.1 degree. The results are shown in Fig.2. The trends of the scan curves matched well with the simulation results and difference of the resonant angle were able to be solved by calibrate the mechanical angles.

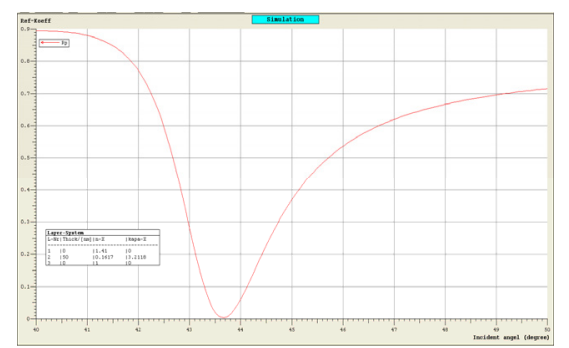

(a)

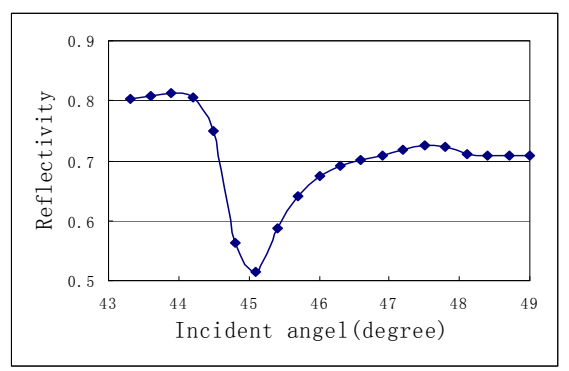

(b)

Fig. 2. Graph of simulation(a) and experiment(b) results of imaging SPR resonant angle in the air

In Fig.3 we tested SPR performance of a right angle glass prism with $\mathrm{RI}$ of 1.51 to compare with the PDMS prism. The resonant angle of glass prism was 44.1 degree that was consistent with numerical calculation. However, the depth of SPR absorption peak for the glass prism only half of the PDMS prism. As a consequence, the sensitivity of the PDMS prism is twice of the glass one if the other conditions did not change. Moreover, the signal noise of the glass prism is higher because the index matching oil would diffuse slightly for more than 2 hours to be stable. That is a great drawback for field rapid use.

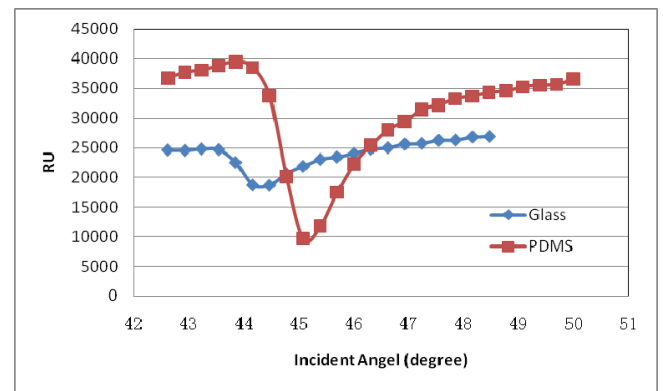

Fig. 3. Performance comparison of PDMS and glass prisms 


\section{Rapid detection of different gas}

Experiment was carried on our self-developed SPR imaging system with PDMS prism. Series 0-5 showed by different colors correspond to six different area on gold surface and they have similar response. The process of the detection is as followed shown in Fig.4: (a) Detect the air as the baseline of other gas. (b) Detect the gas breathed out by person $A$ and the response of the signal has reduced about $6000 \mathrm{RU}$. (c) Detect the air again and the baseline goes back to the beginning of the detection. (d) Repeated detect the gas breathed out by person $A$ for 10 second. (e) Detect the air again (f) Detect the gas breathed out by person $B$ and the response of the signal has reduced about $2000 \mathrm{RU}$. (g) Detect the air again. (h) Detect the gas breathed out by person $\mathrm{C}$ and the response of the signal has reduced about $5000 \mathrm{RU}$. (i) Detect the air again and the signal gets back.

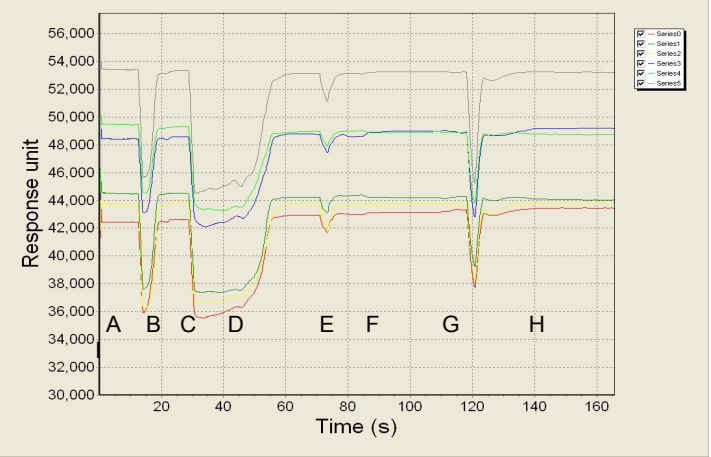

Fig. 4. The detection of different gas.

\section{4. conclusion}

In conclusion, we have made a PDMS prism integrated with four channel flow cell and demonstrated that a microfluidic-based PDMS prism could be used in SPR imaging system and get the SPR angle. We also used the system to test the gas breathed out by different people and the system have a good sensitivity to distinguish different samples. The PDMS prism integrated with multi-channels SPR system makes the low-cost convenient detection possible.

\section{Acknowledgements}

The authors greatly acknowledge the financial support from the Major National Scientific Research Plan under Grant number 2011CB933202. This work is also sponsored by National Science Foundation of China under Grant number 31100820, 61176112, and 60701019. We also thank the financial support from State key laboratory of transducer technology.

\section{References}

[1] S. Scarano, M. Mascini, A. P.F. Turner, M. Minunni. Surface plasmon resonance imaging for affinity-based biosensors. Biosensors and Bioelectronics 25, 957-966(2010)

[2] J. Homola, SS. Yee, G. Gauglitz. Surface plasmon resonance sensors: review. Sensors and Actuators B 54, 3-15(1999)

[3] A. Nooke, U. Beck, A. Hertwig, A. Krause, H. Kruger, V. Lohse, D. Negendank, J. Steinbach. On the application of gold based SPR sensors for the detection of hazardous gases. Sensors and Actuators B 149, 194-198(2010)

[4] M. Varshney, Y. Li, B. Srinivasan, S. Tung. A label-free, microfluidics and interdigitated array microelectrode-based impedance biosensor in combination with nanoparticles immunoseparation for detection of Escherichia coli O157:H7 in food samples. Sensors and Actuators B 128, 99-107( 2007)

[5] Z. Geng, Q. Li, W. Wang, Z. Li. PDMS prismglass optical coupling for surface plasmon resonance sensors based on MEMS technology. Science China 53, 2144 - 2158 (2010)

[6] C.H. Lin, G.B. Lee, Micromachined flow cytometers with embedded etched optic fibers for optical detection. J. Micromech. Microeng. 13, 447 (2003)

[7] A. Llobera, R. Wilkea, S. Buttgenbach. Enhancement of the response of poly(dimethylsiloxane) hollow prisms through air mirrors for absorbance-based sensing. Talanta $75,473-479(2008)$

[8] CC Liu, DF Cui, H Li. A hard-soft microfluidicbased biosensor flow cell for SPR imaging application. Biosensors and Bioelectronics26, 255-261(2010)

[9] D. Cui, H. Cai, J. Wang, Y. Li, J.Liang, C. Liu, Y. Wang, Z. Zheng, Chinese Patent Appl. No. CN 200610066542.4. (2006). 